\title{
CONEXÃO DE SISTEMAS VERTICAIS DE REFERÊNCIA LOCAIS AO SISTEMA GEODÉSICO BRASILEIRO COM BASE EM UM SISTEMA VERTICAL DE REFERÊNCIA GLOBAL
}

\author{
Connection of Local Vertical Reference Systems to Brazilian Geodetic System \\ based on a Global Vertical Reference System
}

\author{
Ruth da Maia Moreira ${ }^{1}$ \\ Silvio Rogério Correia de Freitas ${ }^{1}$

\begin{abstract}
1 Universidade Federal do Paraná - UFPR - Curitiba - Paraná - Brasil. Email:ruthdamaia@gmail.com; sfreitas@ufpr.br
\end{abstract}

\section{Resumo}

Os Modelos Globais do Geopotencial (MGGs), em especial os oriundos da missão GOCE, associados a Modelos Digitais de Altitude (MDAs) com resoluções cada vez melhores, têm possibilitado novas alternativas para a obtenção de altitudes com significado físico. Neste trabalho, propõe-se a realização de um sistema local de altitudes, em uma área onde o Sistema Geodésico Brasileiro (SGB) é deficiente, e vinculá-lo a um Sistema Vertical de Referência Global (SVRG) e ao SGB. Os MGGs foram validados utilizando Referências de Nível associadas com posicionamento GPS. Para modelar o erro de omissão dos MGGs, foi utilizada a técnica Residual Terrain Model (RTM). Os números geopotenciais foram obtidos com a resolução simplificada do Problema do Valor de Contorno da Geodésia (PVCG) na forma fixada. A área de estudos é o Estado do Amapá, que utiliza o Datum Vertical Brasileiro de Santana (DVB-S), e para validação dos MGGs foram utilizadas estações do Estado do Pará, vinculadas ao Datum Vertical Brasileiro de Imbituba (DVB-I). Como resultados, obteve-se números geopotenciais e altitudes físicas consistentes. Na validação dos modelos, o RTM não teve influência significativa. Utilizando o MGG do GOCE, GO_CONS_SPW_R4, obteve-se que o segmento da rede vinculado ao DVB-S está 1,30m acima do segmento vinculado ao DVB-I.

Palavras-chave: Modelos Globais do Geopotencial; Sistema Vertical de Referência Global; Problema do Valor de Contorno da Geodésia; RTM.

\section{Abstract}

Global Geopotential Models (GGMs), specially those provided by GOCE mission, associated with Digital Elevation Models (DEMs) with increasingly higher resolutions, has enable new alternatives for obtaining physical heights. The purpose of this work is the realization of a local height system, in a region with poor data from the Brazilian Geodetic System (BGS), and link it to a Global Vertical Reference System (GVRS). The GGMs were validated using GPS/leveling stations. The omission error of GGMs was modeled by using the Residual Terrain Model (RTM) 
technique. The geopotential numbers were obtained through a simplified solution of the Geodetic Boundary Value Problem (GBVP) in a fixed approach. The study area is the Amapá state, whose altitudes are linked with the Brazilian Vertical Datum of Santana (BVD-S), and for validation of GGMs some stations linked to Brazilian Vertical Datum of Imbituba (BVD-I) in the state of Pará were also used. As a result, geopotential numbers and heights with physical meaning were obtained. In the validation of the models, RTM technique had no significant influence. With the GGM from GOCE, GO_CONS_SPW_R4, it was obtained that the network linked to BVD-S is $1,30 \mathrm{~m}$ above the network linked to BVD-I.

Keywords: Global Geopotential Models; Global Vertical Reference System; Geodetic Boundary Value Problem; RTM.

\section{Introdução}

As altitudes dotadas de significado físico, necessárias às aplicações em obras de engenharia, por exemplo, podem ser determinadas de maneira convencional por técnicas de levantamento como o nivelamento geométrico; estas altitudes são vinculadas a um Datum Vertical (DV), normalmente um marégrafo que fornece o Nível Médio do Mar (NMM) local referido a um determinado período de observações. Porém, os sistemas de altitudes dos países da América do Sul não levaram em consideração a influência da gravidade, mas introduziram, como no caso do Brasil, uma correção teórica da gravidade (correção do não paralelismo das superfícies equipotenciais utilizando o modelo da Terra Normal). Desta forma, o efeito sistemático do Datum e a não consideração da gravidade nos levantamentos gera inconsistências nos sistemas de altitudes, principalmente quando se trata de conexão entre países (De Freitas e Blitzkow, 1999; Sánchez, 2002).

Uma abordagem moderna do problema é possibilitada pela determinação da gravidade por meio dos Modelos Globais do Geopotencial (MGGs) e o conhecimento da topografia local tal como o expresso em Modelos Digitais de Altitude (MDAs), oriundos de plataformas orbitais. Neste contexto, os chamados Sistemas Verticais de Referência Locais (SVRLs) ganharam grande possibilidade de realização com base em redes com melhor adequação às redes nacionais e globais. No caso brasileiro, o vínculo de Sistemas e Redes Verticais Locais ao Sistema Geodésico Brasileiro (SGB) e a um Sistema Vertical de Referência Global (SVRG). Isto decorre justamente das novas possibilidades advindas a partir das ferramentas mais atuais da Geodésia, em particular dos MGGs derivados da missão GOCE (Gravity field and steady-state Ocean Circulation Explorer) em 2012 e 2013, em associação com posicionamento GNSS e o uso de MDAs (Gerlach e Rummel, 2012; Rummel, 2013).

$\mathrm{Na}$ presente pesquisa, a proposta é definir um Sistema Vertical de Referência Local, com sustentação em modelo global consistente conforme preconizado por (Rummel, 2013 e Gerlach e Rummel, 2012), em uma região com deficiência de informação altimétrica da rede oficial do país, ou seja, onde o Sistema Geodésico Brasileiro apresenta uma lacuna. Para isto, foi constituída uma base de dados que possibilitou a realização de testes de aderência na área de estudos, observando a consistência dos referenciais e sistemas de maré permanente. Os modelos globais do geopotencial foram avaliados em termos de sua compatibilidade com uma base geodésica conhecida de forma a validar suas aplicações, e o preenchimento dos vazios espectrais

Bol. Ciênc. Geod., sec. Artigos, Curitiba, v. 22, nํ2, p.232 - 247, abr - jun, 2016. 
(erro de omissão) dos modelos do geopotencial foi efetuado com o emprego da técnica RTM Residual Terrain Model. Os números geopotenciais foram obtidos a partir da solução do tipo Brovar para o Problema do Valor de Contorno da Geodésia (PVCG) na opção do problema vetorial fixo, de maneira simplificada. Foi também estimado o afastamento entre o Datum Vertical Brasileiro de Imbituba (DVB-I) e o Datum Vertical Brasileiro de Santana (DVB-S).

A solução do PVCG neste trabalho será o problema vetorial fixo simples, no qual a aproximação é linear, e as observações são referenciadas à superfície física. São conhecidas as coordenadas geodésicas $\varphi, \lambda$ h e a gravidade $g$, e a incógnita é o geopotencial $W$. O problema é resolvido de acordo com a abordagem de Brovar (Brovar, 1964), usando superfície generalizada do potencial anômalo T. O diferencial neste caso é a independência das linhas de nivelamento; necessita-se apenas de dados gravimétricos com densidade adequada (Heck, 2011). Esta densificação precisa conduzir a uma resolução espacial determinada pela taxa de amostragem, que pode ser estabelecida em função da frequência de Nyquist - a melhor resolução espacial é igual à metade da frequência de amostragem.

Em relação aos Modelos Globais do Geopotencial, foi utilizado na solução do problema um MGG oriundo da missão GOCE, pois os modelos obtidos a partir dos dados gravimétricos do GOCE foram propostos por (Gerlach e Rummel, 2012) para servirem de base ao chamado Sistema Vertical de Referência Global (SVRG).

A realização do SVRG é possibilitada pela combinação de observações maregráficas, altimetria por radar a bordo de satélites, medidas terrestres, marinhas e aéreas de gravidadealém de técnicas espaciais, coordenadas ITRF e nivelamento geométrico (Ihde e Sánchez, 2005). Na atualidade, com o desenvolvimento das missões gravimétricas por satélite, os MGGs têm sido ferramentas essenciais na materialização dos SVRGs.

A modelagem das altas frequências do sinal gravitacional, o que na atualidade pode ser traduzido como a parcela do sinal gravitacional não contemplada pelos MGGs, é efetuada utilizando técnicas de decomposição espectral. Para a obtenção do sinal relativo aos curtos comprimentos de onda, duas técnicas são de interesse relevante: a clássica Decomposição Espectral ou RemoveRestore (RR) e a modelagem residual do terreno- Residual Terrain Model (RTM).

A técnica RTM consiste em calcular os curtos comprimentos de onda (altas frequências do campo da gravidade) não representados pelo MGG, utilizando uma superfície de referência (MDA) associada ao modelo do geopotencial; as massas topográficas acima da superfície são removidas e abaixo são preenchidas (Forsberg e Tscherning, 1981; Forsberg, 1984).

\section{Materiais e Métodos}

A área de estudos abrange parte dos Estados brasileiros do Amapá e do Pará, conforme mostra a Figura 1. Nesta figura também são apresentadas as estações que possuem dados de nivelamento e posicionamento GNSS utilizadas para validação dos MGGs, denominadas de estações GPS/NIV. As estações localizadas no Estado do Amapá têm origem no DVB-S, enquanto as estações do Estado do Pará são vinculadas ao DVB-I. 


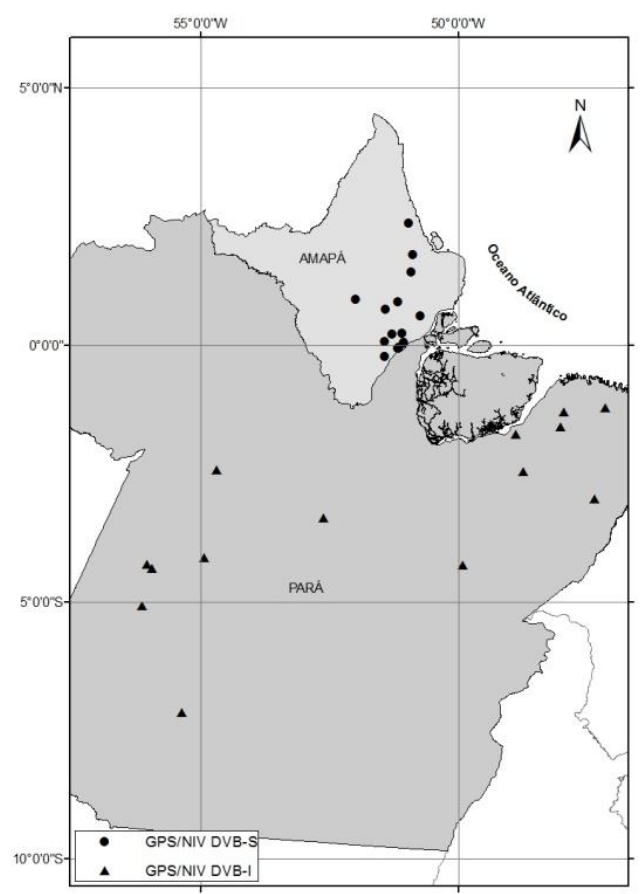

Figura 1: Área de estudos e estações GPS/NIV.

Fonte: Os autores (2014)

Como dados para o trabalho, foram utilizados dois Modelos Globais do Geopotencial somente satélite do GOCE, GO_CONS_SPW_R4 e GO_CONS_TIM_R5, e os modelos combinados EIGEN-6C4 e EGM2008, disponibilizados pelo ICGEM (International Centre for Global Earth Models). As informações referentes aos modelos utilizados podem ser vistas na Tabela 1, na qual nos dados da terceira coluna (Fonte), S indica Satélite, G gravimetria e A altimetria.

Tabela 1: Modelos Globais do Geopotencial utilizados

\begin{tabular}{c|c|c|c|c}
\hline MGG & Ano & Fonte & Grau máx & RE (km) \\
\hline GO_CONS_GCF_2_SPW_R4 & 2014 & S(Goce $)$ & 280 & 71 \\
\hline EIGEN-6C4 & 2014 & S(Goce,Grace,Lageos),G,A & 2190 & 9 \\
\hline GO_CONS_GCF_2_TIM_R5 & 2014 & S(Goce) & 280 & 71 \\
\hline EGM 2008 & 2008 & S(Grace) G,A & 2190 & 9 \\
\hline
\end{tabular}

FONTE: ICGEM (2015) RE - Resolução Espacial

Como forma de validar os modelos do geopotencial, foram utilizadas 17 estações GPS/NIV no Estado do Amapá (Tabela 2) e 15 estações no Estado do Pará (Tabela 3). Nestas tabelas são apresentadas as coordenadas, as altitudes elipsoidais e ortométricas (a rigor normaisortométricas) conhecidas, com seus respectivos desvio-padrão. Para algumas estações, não constam no banco de dados do IBGE o desvio-padrão das altitudes ortométricas. 
Tabela 2: Estações GPS/NIV no Estado do Amapá (DVB-S)

\begin{tabular}{c|c|c|c|c|c|c}
\hline Estação & $\begin{array}{c}\text { Latitude } \\
\left({ }^{\circ}\right)\end{array}$ & $\begin{array}{c}\text { Longitude } \\
\left({ }^{\circ}\right)\end{array}$ & $\begin{array}{c}\text { Altitude } \\
\text { Elipsoidal } \\
\mathbf{h}(\mathbf{m})\end{array}$ & $\begin{array}{c}\text { Desvio } \\
\text { padrão } \\
\mathbf{h}(\mathbf{m})\end{array}$ & $\begin{array}{c}\text { Altitude } \\
\text { Ortométrica } \\
\mathbf{H}(\mathbf{m})\end{array}$ & $\begin{array}{c}\text { Desvio } \\
\text { padrão } \\
\mathbf{H}(\mathbf{m})\end{array}$ \\
\hline 90204 & 0,051434 & $-51,069388$ & $-6,38$ & 0,114 & 16,48 & 0,020 \\
\hline 91215 & $-0,057343$ & $-51,180647$ & $-15,51$ & 0,002 & 7,51 & 0,010 \\
\hline 91240 & $-0,054216$ & $-51,180209$ & $-14,47$ & 0,004 & 8,53 & 0,010 \\
\hline 91241 & $-0,041261$ & $-51,167933$ & $-10,16$ & 0,003 & 12,84 & \\
\hline 90444 & 0,046875 & $-51,071358$ & $-8,14$ & 0,350 & 14,53 & 0,020 \\
\hline 91575 & $-0,060874$ & $-51,160134$ & $-17,56$ & 0,004 & 5,38 & 0,010 \\
\hline 91577 & 0,230618 & $-51,100875$ & 5,16 & 0,089 & 28,33 & \\
\hline 96088 & 0,071905 & $-51,440129$ & $-19,38$ & 0,032 & 3,85 & \\
\hline 96089 & 0,216821 & $-51,292943$ & 7,91 & 0,025 & 31,22 & \\
\hline 96090 & $-0,221959$ & $-51,431898$ & $-14,95$ & 0,070 & 7,85 & \\
\hline 96091 & 0,843016 & $-51,184826$ & 28,79 & 0,026 & 53,11 & \\
\hline 96092 & 1,423701 & $-50,922780$ & 2,84 & 0,025 & 29,25 & \\
\hline 96094 & 1,765378 & $-50,882325$ & $-11,62$ & 0,030 & 15,91 & \\
\hline 96095 & 2,373195 & $-50,963819$ & $-11,17$ & 0,027 & 17,39 & \\
\hline 96096 & 0,576110 & $-50,738378$ & $-9,84$ & 0,033 & 14,34 & \\
\hline 96097 & 0,704020 & $-51,423133$ & 53,94 & 0,035 & 78,28 & \\
\hline 96098 & 0,901443 & $-52,002336$ & 123,58 & 0,042 & 149,11 & \\
\hline
\end{tabular}

FONTE: IBGE (acesso em 2014)

Tabela 3: Estações GPS/Niv no Estado do Pará (DVB-I)

\begin{tabular}{c|c|c|c|c|c|c}
\hline Estação & $\begin{array}{c}\text { Latitude } \\
\left({ }^{\circ}\right)\end{array}$ & $\begin{array}{c}\text { Longitude } \\
\left({ }^{\circ}\right)\end{array}$ & $\begin{array}{c}\text { Altitude } \\
\text { Elipsoidal } \\
\mathbf{h}(\mathbf{m})\end{array}$ & $\begin{array}{c}\text { Desvio } \\
\text { padrão } \\
\mathbf{h}(\mathbf{m})\end{array}$ & $\begin{array}{c}\text { Altitude } \\
\text { Ortométrica } \\
\mathbf{H}(\mathbf{m})\end{array}$ & $\begin{array}{c}\text { Desvio } \\
\text { padrão } \\
\mathbf{H}(\mathbf{m})\end{array}$ \\
\hline 90411 & $-7,142402$ & $-55,382231$ & 208,57 & 0,014 & 224,32 & 0,120 \\
\hline 90448 & $-6,835331$ & $-49,582874$ & 200,26 & 0,013 & 221,83 & 0,100 \\
\hline 95000 & $-3,353709$ & $-52,623018$ & 138,92 & 0,019 & 163,79 & 0,120 \\
\hline 96073 & $-4,272153$ & $-49,925111$ & 65,85 & 0,027 & 89,56 & 0,100 \\
\hline 96072 & $-1,211136$ & $-47,146500$ & 9,78 & 0,020 & 35,28 & \\
\hline 90609 & $-1,297518$ & $-47,949816$ & 19,94 & 0,023 & 45,52 & 0,100 \\
\hline 93239 & $-1,724884$ & $-48,889640$ & $-18,36$ & 0,017 & 6,34 & \\
\hline 93998 & $-2,992504$ & $-47,363271$ & 51,12 & 0,041 & 77,49 & 0,100 \\
\hline 93997 & $-1,582550$ & $-48,016363$ & 3,04 & 0,012 & 28,63 & 0,100 \\
\hline 96069 & $-2,455118$ & $-48,740743$ & $-3,87$ & 0,013 & 22,67 & \\
\hline 93996 & $-5,069668$ & $-56,157462$ & 91 & 0,013 & 108,72 & 0,120 \\
\hline 93693 & $-2,426354$ & $-54,701349$ & 9,1 & 0,036 & 29,92 & \\
\hline 95001 & $-4,124724$ & $-54,951769$ & 97,66 & 0,013 & 118,62 & 0,120 \\
\hline 95003 & $-4,343991$ & $-55,960851$ & 18,3 & 0,017 & 38,25 & 0,120 \\
\hline 95002 & $-4,267674$ & $-56,049324$ & 7,96 & 0,021 & 27,87 & 0,120 \\
\hline
\end{tabular}

FONTE: IBGE (acesso em 2014) 


\subsection{Validação dos MGGs}

Uma forma bastante usual de realizar a validação dos modelos do geopotencial é a comparação direta com dados levantados em campo, normalmente estações que possuem nivelamento e posicionamento GNSS associados.

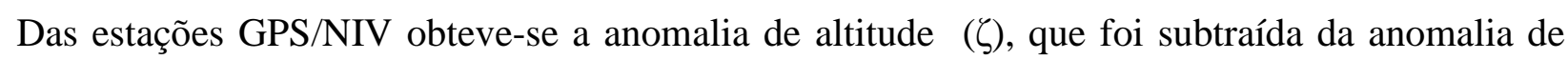
altitude obtida dos MGGs em seus graus máximos do desenvolvimento em séries de funções harmônicas esféricas, resultando nas diferenças das quais é possível inferir o erro absoluto ( $\varepsilon$ absoluto).

$$
\varepsilon_{\text {absoluto }}=\zeta_{\text {GPS/NIV }}-\zeta_{M G G}
$$

Para obter a estimativa do erro relativo, foi calculada a diferença entre $\zeta_{\text {GPS/NIV- }} \zeta_{\text {MGG }}$ e

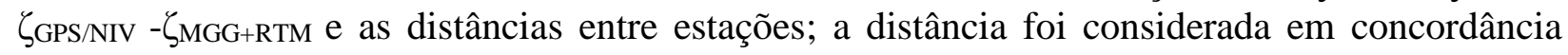
com a resolução espacial dos MGGs. Por exemplo, para o MGG GO_CONS_TIM_R5, que tem resolução de $71 \mathrm{~km}$, foram utilizadas para o cálculo apenas estações cujas distâncias entre si fossem iguais ou maiores que a resolução do modelo.

$$
\varepsilon_{\text {relativo }}=\frac{\Delta \zeta_{G P S / N I V}-\Delta \zeta_{M G G}(\mathrm{~cm})}{D_{i}(\mathrm{~km})}
$$

\subsection{Compatibilização dos sistemas de maré permanente}

As coordenadas ITRFxx e o posicionamento obtido com o sistema GPS estão no sistema livre de maré (tide-free). Em relação ao sistema de altitudes brasileiro, não foram aplicadas correções de maré ao nivelamento, portanto as altitudes normais-ortométricas estão no sistema médio de maré (mean-tide).

É necessário, portanto, realizar a compatibilização dos sistemas de maré aos dados utilizados. As funcionais dos MGGs foram obtidas no sistema livre de maré. Para converter as altitudes normais-ortométricas das estações GPS/NIV do sistema médio de maré para o sistema livre de maré utilizou-se a formulação apresentada por (Tenzer et al., 2010):

$$
H_{\text {tide-free }}=H_{\text {mean-tide }}+\left\{(1+k-h)\left[-0.198\left(\frac{3}{2} \operatorname{sen}^{2} \varphi-\frac{1}{2}\right)\right]\right\}
$$

$k$ e $h$ são os números de Love relativos à maré: $\mathrm{k}=0.3 \mathrm{e} \mathrm{h}=0.6$

$\varphi$ é a latitude geocêntrica

\subsection{Abordagem do PVCG na forma fixada}

Como solução do problema da obtenção de altitudes com significado físico sem dependência de rede de nivelamento, será abordada a solução do tipo Brovar para o PVCG na forma fixada, com a determinação de números geopotenciais baseados na modelagem do potencial anômalo. 
O número geopotencial $C_{P}$ é dado pela diferença entre o geopotencial no geoide e o geopotencial no ponto $P$ considerado. A integral é independente do caminhamento utilizado (Torge, $2001 \mathrm{p}$. 80):

$$
C_{P}=W_{0}-W_{P}=-\int_{P_{0}}^{P} d W=\int g d n
$$

Com esta abordagem, (Heck, 2011) afirma que na solução fixada do PVCG o número geopotencial $C_{P}$ não depende de nivelamento e gravimetria ao longo das linhas de nivelamento, mas é derivado da solução do PVCG fixo para qualquer ponto na superfície terrestre.

Supondo que na região de interesse houvesse informação gravimétrica densa o suficiente (por exemplo, um ponto a cada $100 \mathrm{~km}^{2}$, o que corresponderia a uma resolução espacial de $20 \mathrm{~km}$, considerada a frequência de Nyquist, o que implica em grau/ordem aprox. 1000), a solução completa da técnica Remove-Restore, para a abrangência dos longos e curtos comprimentos de onda do campo da gravidade, poderia ser dada da forma apresentada na Tabela 4.

Tabela 4: Exemplo da solução completa da técnica Remove-Restore

\begin{tabular}{ccccc}
\hline Grau/ordem & 0 a 250 & 250 a 1000 & 1000 a 2159 & 2159 acima \\
\hline Técnica empregada & MGG GOCE & Gravimetria & EGM2008 & MDA \\
\hline
\end{tabular}

Entretanto, na área de estudos existem somente 8 estações que contêm dados de posicionamento GPS associado com gravimetria, e não estão bem distribuídas, concentrando-se apenas em uma parte do Estado do Amapá. Desta forma, não é possível inserir estas observações na solução, uma vez que distorceriam os resultados. Assim sendo, para a obtenção do número geopotencial em uma região isolada, a solução se resumiu na utilização de MGG do GOCE + RTM, pois não foi possível obter o potencial anômalo $\mathrm{T}$ observado, ou seja, baseado em distúrbios observados da gravidade $\delta \mathrm{g}$.

Na aplicação da técnica Remove-Restore, portanto, as altas frequências do campo da gravidade são abrangidas pelo RTM. Tomando como exemplo a Tabela 4, os graus acima da resolução do MGG GOCE são preenchidos pelo RTM. Excluindo-se a parte residual $\left(T_{R E S}\right)$, a formulação da técnica Remove-Restore, considerando o potencial anômalo T, tem a seguinte forma:

$$
T_{P}=T_{M G G}+T_{R T M}
$$

O subíndice $P$ indica que os valores são obtidos para um determinado ponto na superfície física. O potencial anômalo $T$ representa as irregularidades regionais e locais de $W$, correspondendo a aproximadamente $0,1 \%$ do sinal gravitacional real, enquanto o potencial normal $U$ representa cerca de $99,9 \%$ do sinal gravitacional real $W$. Desta forma, o potencial anômalo é dado por (Torge, 2001 p.214):

$$
T_{P}=W_{P}-U_{P}
$$

Da fórmula de Bruns $N=T \gamma /($ Hofmann-Wellenhof e Moritz, 2005 p. 93), obtém-se a fórmula análoga para o cálculo das anomalias de altitude $\zeta$.

$$
\zeta=\frac{T}{\gamma}
$$


Como se conhece o valor da anomalia de altitude obtida do cálculo do RTM e a gravidade normal $\gamma$ - Equações 11 e 12 - pode-se calcular o valor de $T_{R T M}$ da seguinte forma:

$$
T_{R T M}=\zeta_{R T M} * \gamma
$$

A gravidade normal $\gamma$ para uma dada latitude pode ser calculada pela fórmula de Somigliana Equação 9 (Torge, 2001 p. 106).

$$
\gamma=\frac{a \gamma_{e} \cos ^{2} \varphi+b \gamma_{p} \operatorname{sen}^{2} \varphi}{\left(a^{2} \cos ^{2} \varphi+b^{2} \operatorname{sen}^{2} \varphi\right)^{1 / 2}}
$$

Sendo $\gamma_{\mathrm{e}}$ a gravidade normal no equador, $\gamma_{\mathrm{P}}$ a gravidade normal no polo, $a$ o semi-eixo maior do elipsoide de referência e $b$ o semi-eixo menor.

$\mathrm{O}$ valor de $T_{M G G}$ é calculado também pela fórmula de Bruns, aplicando-se os valores de anomalia de altitude obtidos diretamente do MGG.

O potencial normal $U_{P}$ no ponto da superfície física é dado por (Hofmann-Wellenhof e Moritz, 2005 p. 93):

$$
U_{P}=U_{P \prime}+\frac{\partial U_{P \prime}}{\partial n^{\prime}} h
$$

como $U_{P \prime}=U_{0} \mathrm{e} \frac{\partial U_{P^{\prime}}}{\partial n^{\prime}}=-\gamma$,

$$
U_{P}=U_{0}-\gamma h
$$

$\mathrm{U}_{0}$ é o potencial normal, $h$ é a altitude geodésica e $\gamma$ a gravidade normal.

$\mathrm{O}$ potencial normal no elipsoide de referência é uma constante física. Para o SGR80, $\mathrm{U}_{0}=$ 62.636.860,850 m² $\mathrm{s}^{-2}$ (Hofmann-Wellenhof e Moritz, 2005 p. 86). Entretanto, por convenção, faz-se $\mathrm{W}_{0}=\mathrm{U}_{0}$. Será utilizado o valor de $\mathrm{W}_{0}=62.636 .856,0 \pm 0,5 \mathrm{~m}^{2} \mathrm{~s}^{-2}$, divulgado por (Burša et al., 2004) e bastante aceito pela comunidade científica.

\subsubsection{Comparação entre o geopotencial global e local}

Para indicar o geopotencial de acordo com a superfície de referência considerada, são adotadas neste trabalho as seguintes nomenclaturas: $\mathrm{W}_{0}=$ geopotencial global (geoide); $\mathrm{W}_{\mathrm{i}}=$ geopotencial no Datum local (neste caso DVB-I) e $\mathrm{W}_{\mathrm{P}}=$ geopotencial no ponto $\mathrm{P}$ da superfície física. O geopotencial no Datum é dado pela equação:

$$
W_{i D V B}=W_{0}-\gamma_{D V B} \cdot \delta\left(H_{0}\right)_{D V B}
$$

onde $\gamma_{D V B}$ é a gravidade normal no Datum e $\delta H_{0}$ é a discrepância vertical entre o geopotencial do DVB-I e o geopotencial global. (Ferreira et al., 2010) realizaram a determinação da diferença de potencial e chegaram a $\delta W=-0,31 \mathrm{~m} \pm 0,01 \mathrm{~m}$, ou diferença de potencial $\delta W=3,04 \pm 0,08 \mathrm{~m}^{2} \mathrm{~s}^{-2}$, ou seja, o geopotencial no DVB-I está abaixo do geoide global do EGM2008 em $31 \mathrm{~cm}\left(\delta H_{0}=\right.$ $31 \mathrm{~cm})$. Obteve-se assim um valor de geopotencial $W_{i}$ no DVB-I igual a $62.636 .859,04 \mathrm{~m}^{2} \mathrm{~s}^{-2}$.

Na obtenção dos números geopotenciais, $C_{P}$ é o número geopotencial com caráter global, pois relaciona o geoide global $W_{0}$ e o geopotencial $W_{P}$, que tem referência à superfície global por estar diretamente relacionado a um MGG $\left(C_{P}=W_{0}-W_{P}\right)$. Para calcular o número geopotencial local $C_{i}$, foi utilizado o geopotencial $W_{i}$ referente ao Datum de $\operatorname{Imbituba}\left(C_{i}\right.$ IMB $=W_{i}-W_{P}$ IMB $)$. 
Para que o geopotencial $W_{P}$ no ponto $P$ tenha referência ao geoide local, subtrai-se a diferença de geopotencial $\delta W$, obtendo-se o valor de $W_{P}$ IMB.

$$
\begin{aligned}
& C_{\text {IMB }}=W_{i}-W_{P_{I M B}} \\
& W_{P_{I M B}}=U_{P}+T_{P_{I M B}} \\
& T_{P_{I M B}}=T_{M G G}-\delta W
\end{aligned}
$$

Para obter os números geopotenciais com caráter local das estações que têm origem no DVB-S $\left(C_{i} S_{S T N}\right)$ é preciso considerar a discrepância existente entre os dois Data $\left(\delta W_{I M B-S T N}\right)$, determinado neste trabalho e igual a 1,30m para o MGG GO_CONS_SPW_R4.

$$
\begin{gathered}
C_{i S T N}=W_{i}-W_{P S T N} \\
W_{P S T N}=U_{P}+T_{P S T N} \\
T_{P S T N}=T_{M G G}-\delta W-\delta W_{I M B-S T N}
\end{gathered}
$$

Também foi possível, aplicando a Equação 11, obter um valor de geopotencial $W_{0}$ no DVB-S; constatou-se que o geoide local no DVB-S está $0,99 \mathrm{~m}$ acima do geoide global adotado nesta pesquisa. $\mathrm{O}$ valor obtido para $W_{i}$ em Santana foi de $62.636 .846,29 \mathrm{~m}^{2} \mathrm{~s}^{-2}$.

A discrepância entre o Datum Vertical local (geopotencial $W_{i}$ ), e o Datum Vertical global (geopotencial $W_{0}$ ) é dada em termos dos números geopotenciais na forma:

$$
C_{P}-C_{i}=W_{0}-W_{i}=\delta W
$$

Os resultados das discrepâncias em termos dos números geopotenciais são apresentados na Tabela 3, tanto para o DVB-S quanto para o DVB-I.

\section{Resultados}

Antes de proceder à validação dos MGGs, efetuou-se a compatibilização dos sistemas de maré permanente. A conversão do sistema de maré médio de maré para sistema livre de maré resultou em $6,7 \mathrm{~cm}$, ou seja, as altitudes normais-ortométricas foram elevadas em média $6,7 \mathrm{~cm}$; estas altitudes corrigidas foram utilizadas na validação dos modelos.

\subsection{Erro absoluto}

A média das diferenças ( $\left.\zeta_{\mathrm{GPS} / \mathrm{NIV}}-\zeta_{\mathrm{MGG}}\right)$ são mostradas na Figura 2. Verifica-se que a maioria dos modelos tem comportamento semelhante, com diferenças médias de $+0,65 \mathrm{~m}$ para as estações do DVB-S e - $0,68 \mathrm{~m}$ para as estações do DVB-I. Fazendo a diferença das médias entre as estações com vínculo no DVB-S e as estações com vínculo no DVB-I, obtém-se a estimativa de afastamento entre os dois Data. Considerando o MGG GO_CONS_SPW_R4 até o grau 280, a diferença é de $1,30 \mathrm{~m}$. Incorporando a solução RTM, o afastamento é de $1,32 \mathrm{~m}$. O resultado obtido confirma a diferença existente entre os dois Data Verticais, conforme detectado por (Montecino, 2011). 
É importante ressaltar que nas diferenças encontradas estão embutidas incertezas inerentes à precisão da própria rede de nivelamento, à limitação da precisão do posicionamento GNSS, à resolução alcançada pelo MGG, entre outros; outro fator relevante é que no Estado do Amapá os dados de nivelamento apresentam inconsistências, pois a rede de nivelamento não forma circuitos e portanto as altitudes não são ajustadas.

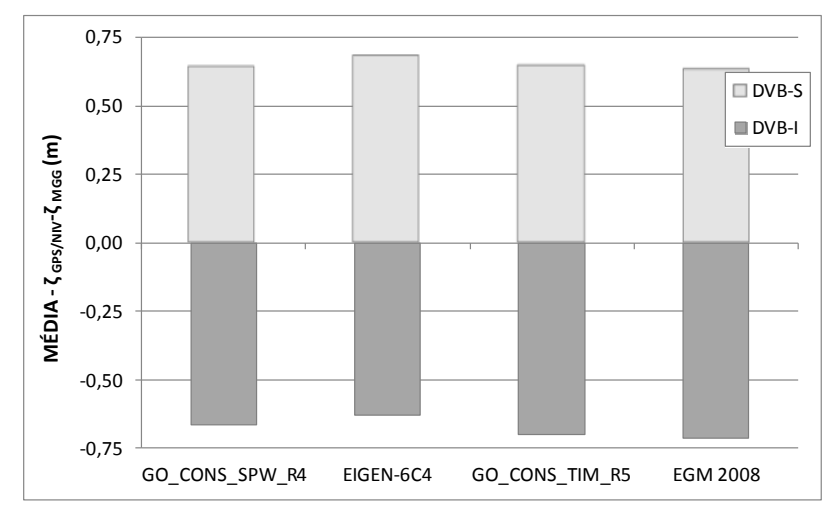

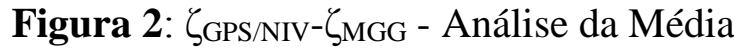

A qualidade dos modelos é avaliada com o erro médio quadrático (RMS - Root Mean Square). Para que o RMS reflita a qualidade real dos modelos, deve-se retirar o efeito sistemático, traduzido pela média das diferenças $\zeta_{\mathrm{GPS} / \mathrm{NIV}}-\zeta_{\mathrm{MGG}}$. Desta forma, tem-se que a precisão dos modelos $(p)$ é igual ao efeito sistemático $(\overline{\mathrm{e}})$, calculado com a diferença de cada valor em relação à média, subtraído do erro absoluto (e):

$$
p=e-\bar{e}
$$

O RMS para cada um dos Data, isento do efeito sistemático, é apresentado na Figura 3.

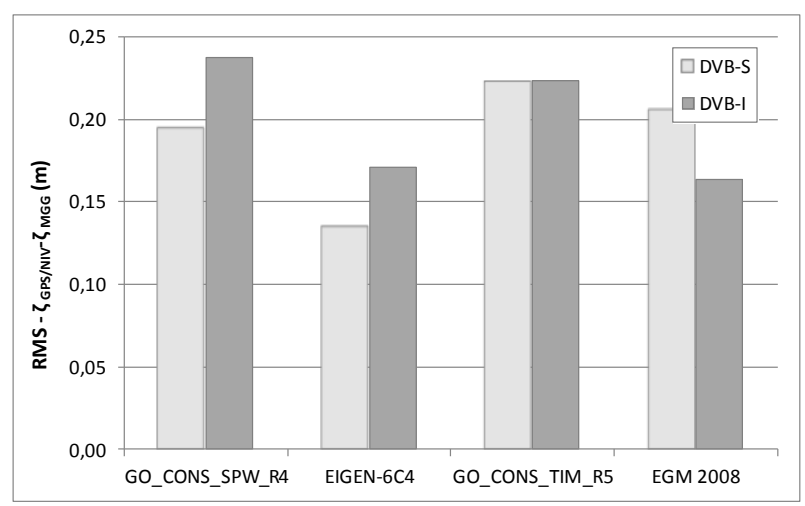

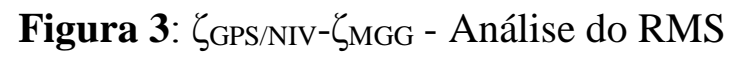




\subsection{Erro relativo}

Na Tabela 5 são apresentados os erros relativos, considerados os graus máximos de cada MGG, para cada um dos Data. Foram consideradas estações aleatórias, com distâncias entre si superiores à resolução espacial dos modelos globais.

As mesmas observações descritas na seção 4.1.1 a respeito das incertezas existentes nos levantamentos e nos MGGs devem ser consideradas na análise do erro relativo.

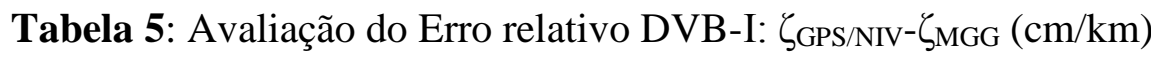

\begin{tabular}{|c|c|c|c|c|c|c|c|c|c|c|c|}
\hline Estações & 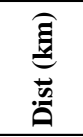 & $\begin{array}{c}\text { GO_CONS } \\
\text { SPW R4 }\end{array}$ & $\begin{array}{c}\text { EIGEN } \\
6 \mathrm{C} 4\end{array}$ & $\begin{array}{c}\text { GO_CONS } \\
\text { TIM R5 }\end{array}$ & $\begin{array}{c}\text { EGM } \\
2008\end{array}$ & Estações & 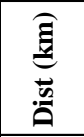 & \begin{tabular}{|c} 
GO_CONS \\
SPW R4
\end{tabular} & $\begin{array}{c}\text { EIGEN } \\
\text { 6C4 }\end{array}$ & $\begin{array}{c}\text { GO_CONS } \\
\text { TIM R5 }\end{array}$ & $\begin{array}{c}\text { EGM } \\
2008\end{array}$ \\
\hline DVB-S & & & & & & DVB-I & & & & & \\
\hline 90204 a 96097 & 82,2 & 0,541 & 0,386 & 0,475 & 0,510 & 93239 a 96069 & 82,4 & 1,200 & 0,348 & 1,104 & 0,267 \\
\hline 96088 a 96096 & 95,9 & 0,221 & $-0,155$ & 0,537 & 0,008 & 90609 a 96072 & 89,9 & 0,139 & 0,389 & $-0,049$ & 0,127 \\
\hline 96092 a 96095 & 105,1 & 0,163 & 0,234 & 0,371 & 0,004 & 95001 a 93996 & 169,7 & $-0,036$ & $-0,104$ & $-0,011$ & 0,066 \\
\hline 96090 a 96091 & 120,9 & 0,039 & 0,063 & 0,097 & 0,292 & 93997 a 93998 & 172,0 & $-0,225$ & $-0,034$ & $-0,218$ & $-0,208$ \\
\hline 96097 a 96094 & 131,9 & $-0,026$ & $-0,047$ & 0,021 & 0,153 & 95003 a 93693 & 254,0 & 0,002 & 0,023 & $-0,079$ & 0,137 \\
\hline 96089 a 96092 & 139,6 & 0,055 & $-0,066$ & 0,047 & 0,203 & 95000 a 96073 & 316,4 & $-0,050$ & 0,118 & $-0,104$ & 0,121 \\
\hline 91241 a 96098 & 139,6 & 0,328 & 0,200 & 0,322 & 0,202 & 95002 a 90411 & 326,3 & $-0,041$ & $-0,032$ & $-0,059$ & 0,104 \\
\hline 91577 a 96095 & 237,4 & 0,148 & 0,101 & 0,204 & 0,145 & 96072 a 96073 & 458,3 & $-0,084$ & \begin{tabular}{|l|}
$-0,069$ \\
\end{tabular} & $-0,075$ & 0,002 \\
\hline
\end{tabular}

Em geral, os MGGs apresentaram um bom desempenho relativo. Destaca-se que o EIGEN-6C4 teve o melhor resultado para a maioria das linhas de base consideradas, provavelmente porque este modelo possui os dados de gradiometria do GOCE, além dos dados de campo.

Para as estações vinculadas ao DVB-I, constata-se que para a menor linha de base $(82,4 \mathrm{~km})$, o erro relativo é maior, chegando a mais que $1 \mathrm{~cm} / \mathrm{km}$ para os MGGs somente-satélite. Entretanto, em sua maioria, os MGGs também têm erros relativos aceitáveis.

\subsection{Resultado do RTM}

As elevações RTM foram construídas como a diferença entre as elevações do GMRT (Global Multi-Resolution Topography), que tem resolução espacial de $30 \mathrm{~m}$ no terreno e $100 \mathrm{~m}$ nas áreas oceânicas, e um MDA desenvolvido em séries de funções harmônicas esféricas, o ETOPO1, com resolução de 1' de arco e desenvolvido até o grau e ordem 2250. O RTM foi calculado truncando a topografia do ETOPO1 e os MGGs nos graus e ordem 180, 210, 250, 360 e graus máximos dos modelos globais considerados. Na Tabela 6 são mostrados os resultados para os diferentes graus e o efeito RTM médio para as estações em cada um dos Data. Observa-se que o efeito, em geral, é milimétrico, sendo que os maiores valores obtidos são para o grau e ordem 180 de desenvolvimento em séries de funções harmônicas esféricas. 
Tabela 6: Resultado do RTM - Média para as estações

\begin{tabular}{c|c|c}
\hline \multirow{2}{*}{$\begin{array}{c}\text { Grau/ordem } \\
\text { MGG }\end{array}$} & \multicolumn{2}{|c}{ Média dos efeitos RTM (m) } \\
\cline { 2 - 3 } & DVB-S & DVB-I \\
\hline 2190 & $-0,0030$ & $-0,0026$ \\
\hline 360 & $-0,0049$ & $-0,0328$ \\
\hline 280 & $-0,0547$ & $-0,0421$ \\
\hline 250 & $-0,0468$ & 0,0058 \\
\hline 210 & 0,0025 & $-0,0515$ \\
\hline 180 & $-0,1825$ & $-0,1170$ \\
\hline
\end{tabular}

Na análise da qualidade dos modelos, o efeito do RTM foi incorporado ao RMS para verificar qual a contribuição do RTM, retirado o efeito sistemático. De forma geral, a adição do efeito RTM à diferença $\zeta_{\mathrm{GPS} / \mathrm{NIV}}-\zeta_{\mathrm{MGG}}$ não melhorou a discrepância existente entre as duas técnicas, provavelmente devido à topografia plana da região de estudos, com pouca variação no relevo. Em regiões de relevo acentuado, há experimentos que comprovam a eficácia do RTM no preenchimento das feições relativas à topografia (Hirt et al., 2010).

Para algumas estações a aplicação do efeito do terreno residual não teve o resultado esperado, ou seja, houve piora na solução, provavelmente devido às imperfeições do modelo batimétrico disponível para as análises. Nas estações em que houve melhora, esta não foi significativa.

\subsection{Obtenção dos números geopotenciais}

Para a obtenção dos números geopotenciais foram aplicadas as formulações e a metodologia apresentados na Seção 3.4. O MGG utilizado foi o somente-satélite do GOCE, GO_CONS_SPW_R4, do ano de 2014. Os testes de validação dos MGGs não mostraram diferenças significativas entre os modelos, porém foi escolhido este MGG por ser o mais recente do GOCE e devido à metodologia de cálculo empregada, baseada em grades. Destaca-se que o erro de omissão deste modelo é muito próximo ao existente para os demais modelos do GOCE $(30 \mathrm{~cm})$, uma vez que o erro de omissão depende principalmente do grau máximo dos harmônicos esféricos considerados (Reguzzoni, 2015 - comunicação pessoal). O MGG foi utilizado no grau e ordem 210 de desenvolvimento em séries de funções harmônicas esféricas, pois se expandido até o grau 280, não existe melhora significativa na modelagem do campo da gravidade pelo modelo.

Os resultados para todas as estações são mostrados na Tabela 7. A unidade de medida dos números geopotenciais é o kilogal $\mathrm{x}$ metro (kGal.m), que equivale a $10 \mathrm{~m}^{2} \mathrm{~s}^{-2}$; para derivar altitudes a partir dos números geopotenciais, divide-se por um valor da gravidade que depende do tipo de altitude que se deseja determinar. Na referida tabela é apresentada também a diferença obtida nos números geopotenciais com vínculo no geopotencial global e local $\left(\mathrm{C}_{\mathrm{P}}-\mathrm{C}_{\mathrm{i}}\right)$.

A média calculada pelas diferenças entre os números geopotenciais referidos ao Datum Vertical local $\left(C_{i}\right)$ e os com caráter global $\left(C_{P}\right)$, derivados da solução do PVCG, reflete a discrepância existente entre a rede de altitudes oficial do país e o sistema proposto, materializado pelo GOCE ( $\delta W=W_{0^{-}} W_{i}$ ou, analogamente, $\delta W=C_{P^{-}} C_{i}$ ), para ambos os Data Verticais de Santana e Imbituba.

Na comparação entre $C_{P}$ e $C_{i}$, obteve-se um afastamento médio $(\delta W)$ de $-0,56 \mathrm{~m}$ para as estações com vínculo no DVB-I, e de $+0,72 \mathrm{~m}$ para as estações com vínculo no DVB-S. Este resultado é 
coerente com a solução $\zeta_{G P S / N I V}-\zeta_{M G G}$ (erro absoluto, Seção 4.1), com a qual obteve-se um offset de 1,30m entre os dois DVs. Com a solução dos números geopotenciais, resulta uma discrepância de $1,31 \mathrm{~m}$. Salienta-se que no afastamento $\delta W$ obtido estão inseridos o erro de omissão do MGG usado na solução, além de efeitos locais provenientes do cálculo do $W_{i}$. Neste caso, o geopotencial local $W_{i}$ está associado ao EGM2008, cuja acurácia não é uniforme globalmente, mas depende dos dados terrestres de altimetria e gravimetria disponíveis.

Tabela 7: Números geopotenciais obtidos

\begin{tabular}{c|c|c|c}
\hline $\begin{array}{c}\text { Estação } \\
\text { DVB-S }\end{array}$ & $\begin{array}{c}\mathbf{C}_{\mathbf{P}}=\mathbf{W}_{\mathbf{0}}-\mathbf{W}_{\mathbf{P}} \\
(\mathbf{k G a l . m})\end{array}$ & $\begin{array}{c}\mathbf{C}_{\mathbf{i}}=\mathbf{W}_{\mathbf{i}}-\mathbf{W}_{\mathbf{P S T N}} \\
(\mathbf{k G a l . m})\end{array}$ & $\begin{array}{c}\mathbf{C}_{\mathbf{P}}-\mathbf{C}_{\mathbf{i}} \\
(\mathbf{k G a l . m})\end{array}$ \\
\hline 90204 & 170,181 & 163,513 & 6,668 \\
\hline 91215 & 79,351 & 72,262 & 7,089 \\
\hline 91240 & 89,561 & 82,463 & 7,099 \\
\hline 91241 & 131,869 & 124,750 & 7,120 \\
\hline 90444 & 152,901 & 145,677 & 7,224 \\
\hline 91575 & 59,261 & 52,216 & 7,045 \\
\hline 91577 & 285,486 & 277,782 & 7,703 \\
\hline 96088 & 44,937 & 37,012 & 7,925 \\
\hline 96089 & 313,063 & 304,920 & 8,143 \\
\hline 96090 & 83,612 & 76,747 & 6,865 \\
\hline 96091 & 527,364 & 520,665 & 6,699 \\
\hline 96092 & 292,073 & 284,844 & 7,229 \\
\hline 96094 & 161,567 & 153,681 & 7,887 \\
\hline 96095 & 176,088 & 169,396 & 6,692 \\
\hline 96096 & 145,405 & 138,945 & 6,460 \\
\hline 96097 & 771,973 & 764,978 & 6,995 \\
\hline 96098 & $1.462,889$ & 1455,846 & 7,043 \\
\hline \multicolumn{4}{|c}{ MÉDIA } \\
\cline { 2 - 4 }
\end{tabular}

\begin{tabular}{c|c|c|c}
\hline $\begin{array}{c}\text { Estação } \\
\text { DVB-I }\end{array}$ & $\begin{array}{c}\mathbf{C}_{\mathbf{P}}=\mathbf{W}_{\mathbf{0}}-\mathbf{W}_{\mathbf{P}} \\
(\mathbf{k G a l . m})\end{array}$ & $\begin{array}{c}\mathbf{C}_{\mathbf{i}}=\mathbf{W}_{\mathbf{i}}-\mathbf{W}_{\mathbf{P I M B}} \\
(\mathbf{k G a l . m})\end{array}$ & $\begin{array}{c}\mathbf{C}_{\mathbf{P}}-\mathbf{C}_{\mathbf{i}} \\
(\mathbf{k G a l . m})\end{array}$ \\
\hline 90411 & 2190,046 & 2195,390 & $-5,343$ \\
\hline 95000 & 1594,495 & 1598,246 & $-3,752$ \\
\hline 96073 & 874,609 & 879,846 & $-5,238$ \\
\hline 96072 & 337,817 & 342,159 & $-4,343$ \\
\hline 90609 & 438,495 & 444,175 & $-5,680$ \\
\hline 93239 & 62,804 & 69,534 & $-6,730$ \\
\hline 93998 & 749,847 & 757,235 & $-7,388$ \\
\hline 93997 & 273,389 & 280,622 & $-7,232$ \\
\hline 96069 & 210,937 & 215,648 & $-4,711$ \\
\hline 93996 & 1060,001 & 1064,245 & $-4,245$ \\
\hline 93693 & 285,963 & 292,545 & $-6,582$ \\
\hline 95001 & 1153,856 & 1158,988 & $-5,132$ \\
\hline 95003 & 366,553 & 372,541 & $-5,988$ \\
\hline 95002 & 264,961 & 271,263 & $-6,302$ \\
\hline \multicolumn{4}{|r}{} \\
\cline { 2 - 4 }
\end{tabular}

O afastamento maior obtido para as estações com vínculo no DVB-S, quando comparado ao obtido para as estações com vínculo no DVB-I, pode ser atribuído às inconsistências existentes nas estações do SGB no Estado do Amapá.

\subsubsection{Altitudes obtidas a partir dos números geopotenciais}

A partir dos números geopotenciais pode-se derivar diferentes tipos de altitudes físicas, dependendo do tipo de gravidade empregada (e.g gravidade real, gravidade normal, gravidade média). De acordo com os dados disponíveis, e a partir dos números geopotenciais calculados, obteve-se as altitudes dinâmicas e normais para todas as estações da área de estudo. Não é possível obter as altitudes ortométricas, pois estas requerem a formulação de hipóteses simplificativas sobre a distribuição das massas internas da terra. Desta forma, calculou-se as altitudes de Helmert para 8 estações com vínculo no DVB-S e 2 estações com vínculo no DVB-I, pois somente estas estações possuem valores de gravidade observada (Tabela 8). Utilizou-se a redução de Poincaré-Prey para obter o valor de gravidade média $\left(g_{m}\right)$. 
Tabela 8: Altitudes calculadas a partir dos números geopotenciais

\begin{tabular}{|c|c|c|c|c|c|c|c|}
\hline $\begin{array}{c}\text { Estação } \\
\text { DVB-S }\end{array}$ & $\begin{array}{l}\mathrm{H}^{\mathrm{DIN}} \\
(\mathrm{m})\end{array}$ & $\begin{array}{l}\mathrm{H}^{\mathrm{N}} \\
(\mathrm{m})\end{array}$ & $\begin{array}{c}\mathrm{H}^{\text {HELM }} \\
(\mathrm{m})\end{array}$ & $\begin{array}{c}\text { Estação } \\
\text { DVB-I }\end{array}$ & $\begin{array}{l}\mathrm{H}^{\mathrm{DIN}} \\
(\mathrm{m})\end{array}$ & $\begin{array}{l}\mathrm{H}^{\mathrm{N}} \\
(\mathrm{m})\end{array}$ & $\begin{array}{c}\mathrm{H}^{\mathrm{HELM}} \\
(\mathrm{m})\end{array}$ \\
\hline 90204 & 16,674 & 16,719 & & 90411 & 223,878 & 224,452 & \\
\hline 91215 & 7,369 & 7,389 & 7,388 & 95000 & 162,983 & 163,411 & \\
\hline 91240 & 8,409 & 8,432 & 8,431 & 96073 & 89,723 & 89,958 & 89,928 \\
\hline 91241 & 12,722 & 12,755 & & 96072 & 34,892 & 34,984 & \\
\hline 90444 & 14,856 & 14,895 & & 90609 & 45,295 & 45,415 & \\
\hline 91575 & 5,325 & 5,339 & 5,339 & 93239 & 7,091 & 7,110 & \\
\hline 91577 & 28,327 & 28,402 & 28,398 & 93998 & 77,220 & 77,423 & \\
\hline 96088 & 3,774 & 3,784 & 3,784 & 93997 & 28,617 & 28,692 & \\
\hline 96089 & 31,095 & 31,177 & 31,172 & 96069 & 21,991 & 22,049 & 22,048 \\
\hline 96090 & 7,826 & 7,847 & 7,847 & 93996 & 108,528 & 108,810 & \\
\hline 96091 & 53,096 & 53,236 & & 93693 & 29,833 & 29,911 & \\
\hline 96092 & 29,047 & 29,124 & & 95001 & 118,189 & 118,499 & \\
\hline 96094 & 15,672 & 15,713 & & 95003 & 37,990 & 38,090 & \\
\hline 96095 & 17,274 & 17,320 & & 95002 & 27,662 & 27,735 & \\
\hline 96096 & 14,169 & 14,207 & 14,206 & & & & \\
\hline 96097 & 78,010 & 78,216 & & & & & \\
\hline 96098 & 148,462 & 148,854 & & & & & \\
\hline
\end{tabular}

\section{Conclusões e recomendações}

A validação dos modelos do geopotencial com base em estações GPS/NIV mostrou que, em geral, os resultados foram similares para todos os MGGs. Em termos da média, as diferenças

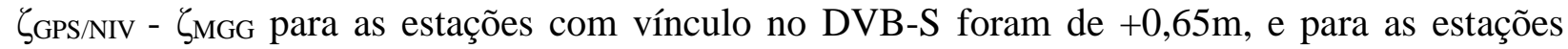
relativas ao DVB-I estas diferenças foram de $-0,68 \mathrm{~m}$. Desta forma, considerando o MGG somente satélite do GOCE, GO_CONS_SPW_R4, pode-se estimar que a rede vinculada ao DVB-S tem sua origem $1,30 \mathrm{~m}$ acima do segmento vinculado ao DVB-I.

Em relação à aplicação da técnica RTM para o preenchimento dos comprimentos de onda não abrangidos pelos MGGs, em geral o RTM não teve contribuição significativa; este comportamento pode ser justificado pelo relevo da área de estudos, praticamente sem variações acentuadas e com altitudes próximas ao nível do mar.

A obtenção dos números geopotenciais no contexto do PVCG na forma fixa mostrou-se bastante adequada, apesar da simplificação da solução devido à falta de dados gravimétricos. A metodologia proposta pode ser aplicada com sucesso principalmente em regiões com escassez de dados de nivelamento. Como não foi possível desenvolver a solução completa do PVCG por falta de dados na área de estudo, recomenda-se a aplicação da solução completa utilizando a gravimetria que está sendo executada pelo Projeto Amapá - Revitalização das Redes Geodésicas do Estado do Amapá, quando do término deste. 
O emprego dos números geopotenciais é vantajoso porque a partir destes é possível derivar diferentes tipos de altitudes, dependendo das necessidades e das convenções estabelecidas. No contexto do SIRGAS, por exemplo, o princípio básico estabelecido pelo Grupo de Trabalho III Datum Vertical - é o cálculo dos números geopotenciais referidos à mesma superfície equipotencial $W_{0}$, e a partir daí cada país deriva altitudes físicas de acordo com suas necessidades.

Os números geopotenciais calculados com caráter global estão referenciados a um MGG oriundo do GOCE, que tem sido apontado para servir de base a um SVRG e também na questão da unificação de sistemas de altitudes. Conclui-se assim a eficácia da utilização dos números geopotenciais no contexto do PVCG para definição e realização de um sistema de altitudes local, em local remoto sem rede de nivelamento existente, vinculado ao SGB e ao SVRG.

\section{REFERÊNCIAS BIBLIOGRÁFICAS}

Brovar, Vsevolod V. "On the solutions of Molodensky's boundary value problem". Bulletin géodésique. v. 72, Issue 1, p 167-173, June 1964.

Burša, M., Kenyon, S., Kouba, J., Šíma, Z., Vatrt, V., Vojtíšková, M. "A global vertical reference frame based on four regional vertical datums". Studia Geophysica et Geodaetica. v. 48, p. 493 - 502, Praga, 2004.

De Freitas, Silvio. R. C., e Blitzkow, Denizar. "Altitudes e Geopotencial". IGeS Bulletin N.9 International Geoid Service, p. 47 - 62, Milan, June 1999.

Ferreira, Vagner G., De Freitas, Silvio R.C. e Heck, Bernhard. "Determinação da diferença de geopotencial do Datum Vertical Brasileiro relativa ao Earth Gravity Model 2008". Revista Brasileira de Cartografia $\mathrm{n}^{\circ}$ 62/03, 2010.

Forsberg, Rene. "A study of terrain reductions, density anomalies and geophysical inversion methods in gravity field modelling". The Ohio State University, Scientific Report $\mathrm{n}^{\circ}$, April 1984.

Forsberg, Rene. and Tscherning, Carl C. "The Use of Height Data in Gravity Field Approximation by Collocation". Journal of Geophysical Research, v. 86, nº 9 , p. 7843-7854, September 10, 1981.

Gerlach, Christian, and Rummel, Reiner. "Global height system unification with GOCE: a simulation study on the indirect bias term in the GBVP approach". Journal of Geodesy; Springer, 2012.

Heck, Bernhard. "A Brovar-Type Solution Of The Fixed Geodetic Boundary Value Problem". Studia Geophysica et Geodaetica. v. 55, Issue 3, p. 441-454, July 2011.

Hirt, Christian., Featherstone, W.E., and Marti, U. "Combining EGM2008 and SRTM/DTM2006.0 residual terrain model data to improve quasi-geoid computations in mountainous areas devoid of gravity data". Journal of Geodesy. 84:557-567. doi 10.1007/s00190-010-0395-1. Springer-Verlag, 2010.

Hofmann-Wellenhof, B., and Moritz, H. Physical Geodesy. Springer WienNewYork, 2005.

IBGE - Instituto Brasileiro de Geografia e Estatística - Banco de Dados Geodésicos. Acesso em 2014. http://www.ibge.gov.br/home/geociencias/geodesia/sgb.shtm 
ICGEM - International Centre for Global Earth Models - Calculation of Gravity Field Functionals on Ellipsoidal Grids. Acesso em 2015. http://icgem.gfz-potsdam.de/ICGEM/.

Ihde, Johannes, and Sánchez, Laura. "A unified global height reference system as a basis for IGGOS". Journal of Geodinamics. v. 40, Issues 4-5, p. 400-413, November-December 2005.

Montecino, Henry. "Proposta de alternativas para conexão dos Data Verticais Brasileiros de Imbituba e Santana". Dissertação de mestrado, Universidade Federal do Paraná, Curitiba, 2011.

Reguzzoni, Mirko. Comunicação pessoal, 2015.

Rummel, Reiner. GIM International Interview, February, 2013.

Sánchez, Laura. "Untersuchung Physikalisher Höhen In Kolumbien" - Determinación de Alturas Físicas En Colombia (Tradução). Institut Für Planetare Geodäsie Technische Universität Dresden. Dresden, November 2002.

Tenzer, Robert, Vatrt, V., Abdalla, A. and Dayoub, N. "Assessment of the LVD offsets for the normal-orthometric heights and different permanent tide systems - a case study of New Zealand". Appl. Geomat. 3: 1 - 8. doi 10.1007/s12518-010-0038-5. Springer, 2010.

Torge, Wofgang. Geodesy. 3rd completely rev. and extended ed. Berlin; New York: Walter de Gruyter, 2001.

Recebido em junho de 2015.

Aceito em setembro de 2015. 